\title{
Linear Feature Extraction from SAR Images based on the modified LSD Algorithm
}

\author{
TAN Xi, ZHAO Lingjun, SU Yi \\ School of Electronic Science and Engineering, NUDT \\ Changsha, China
}

\begin{abstract}
This paper proposes a new approach for linear feature from SAR images based on the modified LSD algorithm. This approach consists of three steps: computing the information of the edge, extracting the lines and the validating the lines. First, the ROEWA detector and the Gabor filter are used to extract the information of the edge, which solves the problem that the LSD algorithm cannot extract the edge and calculate the orientations accurately in SAR images. Second, the points that have the similar orientations can be grouped by region growing to acquire the line-support regions. Then we use rectangles to fit the parameters of the related lines. Finally, using the Helmholtz principle to validate the rectangles and exclude the false lines. The experiment results show that the modified approach can accurately extract linear feature from SAR images. Compared with the classical method of phase group, the proposed method can compute faster and acquire more reliable performance.
\end{abstract}

Index Terms - SAR image, linear feature extraction, LSD, Gabor filter, the Helmholtz principle.

\section{INTRODUCTION}

Synthetic aperture radar (SAR) has been widely used in military reconnaissance and civilian remote sensing exploration with its all-time and all-weather imaging capability and its high spatial resolution. Artificial airports, bridges, roads and other high-value objects are important targets in the SAR image interpretation. Analysis of such linear feature targets mainly depends on the linear feature extraction technology which supports a high-level recognition and information extraction. With the image resolution improving, it is one of the key issues for the SAR image interpretation that extracts the linear features quickly and accurately from mass of data and the complex scene.

Linear feature extraction usually relies on a two-step process: edge detection and line grouping. A lot of classical algorithms are applied for optical image [1-4]. In terms of edge detection, there are many detection operators based on gradient information, such as Sobel operator, Canny operator and so on. In the terms of line grouping, the classical methods are most based or improved on the Hough transform, phase grouping and so on. For SAR image linear feature extraction, because of the multiplicative speckles produced by coherent imaging mechanics different from additive noise in the optical image, the traditional detection operators based on gradient information do not have the CFAR characteristics and often lead to many false edge

This work was supported by National Natural Science

Foundation of China under Grant 61201338 detections. Therefore the pixel-level edge detection methods suitable for optical image often cannot be directly applied for SAR image. For the line grouping, the classical methods and their improved algorithm can be considered. In particular the LSD (Fast Line Segment Detector) [5] [6] algorithm proposed by Grompone et al. in recent years is a new linear feature extraction method. The LSD algorithm first calculates the gradient magnitude and gradient direction of each pixel in images using optics differential method. Then it improves the phase Group [7] to extract candidate segments faster, and finally applies the method [8] [9] proposed by Desolneux which is based on a general perception principle, the Helmholtz to control the number of false detections. In a word, the LSD algorithm extracts linear features fast, accurately, has small amount of false detections and so on.

Despite the LSD algorithm exhibits excellent performance and great potential for application, it is still just a method for the optical image. The purpose of this article is to design a new method of linear feature extraction based on the modified LSD algorithm for the SAR image. The main idea is to obtain the edge information by ROEWA (Ratio of Exponentially Weighted averages) [10] operator and Gabor filter [11] which solve the problem that the LSD algorithm cannot acquire the edge and estimate the edge orientation accurately in a SAR image. Successively, region growing is utilized to collect the pixels which share the same orientation up to a certain precision. The growing regions is defined as a line-support region and then described by rectangular approximation (namely the candidate line segment). Finally, the application of the Helmholtz principle [8] validates candidate line segments and removes the false. The experiment results indicate that the modified LSD algorithm can accurately extract linear features in the SAR image. In addition, compared with the phase of the classic organizational methods, this method shows stronger antiinterference ability, faster computing speed and a stronger practical value.

\section{PROCESSES OF THE LINEAR FEATURE EXTRACTION BASED ON MODIFIED LSD ALGORITHM}

The processes of the proposed method in this article include three parts: the acquisition to the edge information, the extraction of candidate line segments and the line segment validation, as shown in Fig. 1. In the section of acquisition to edge information, the Frost filter [12], the 
ROEWA operator and the Gabor filter are, respectively, used to suppress speckle, detect the edge points and estimate the directions of edge points. In the section of candidate line segment extraction, in order to accelerate the speed of the candidate segment extraction, the edge points are listed in descending order according to the edge intensity. The list gives priority to edge points with higher intensity as seeds in the search of line-support region. In succession, candidate line segments are described by rectangular approximation of the region with respect to the line-support region and the geometric correction is carried out later. Finally, the application of a general perception principle, the Helmholtz principle [8] validates the candidate line segments to filter out the meaningful line segments and eliminate the false ones. The processes are shown in Fig.1.

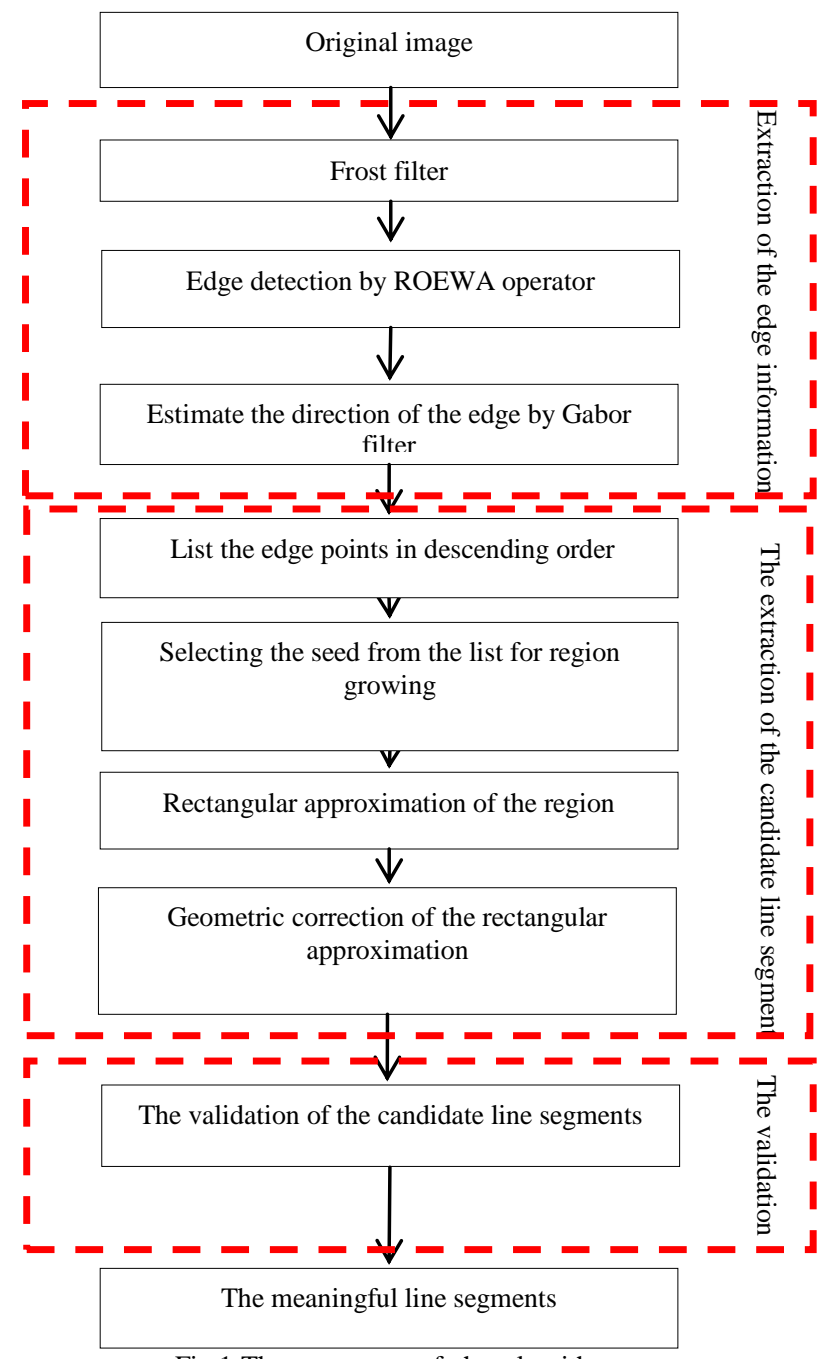

Fig.1.The processes of the algorithm

\section{EDGE INFORMATION ACQUISITION}

\section{A. Edge detection}

Edge detection is the first condition of the follow-up line grouping. SAR image has a lot of speckle that the statistical distribution is different from the additive Gaussian noise in the optical image. The edge detection method for additive Gaussian noise in the LSD algorithm cannot be applied to the SAR image directly. ROEWA operator is the classic algorithm of edge detection in SAR images, which has CFAR properties, edge positioning accurately, few false edges, anti-fracture, etc. So ROEWA operator achieves great outcome to detect the edge in SAR images.

\section{B. Estimation of the edge direction by the Gabor filter}

Gabor filter is the product of a Gaussian which times a complex exponential representing harmonic modulation [13].Gabor filter has excellent directional characteristics and can estimate accurately the direction of the edge in the SAR image. Daugman gives the 2D Gabor function [14]:

$$
g_{\theta}(x, y)=\exp \left\{-\frac{1}{2}\left[\frac{\left(x-u_{x}\right)^{2}}{\lambda \sigma_{x}^{2}}+\frac{\left(y-u_{y}\right)^{2}}{\lambda \sigma_{y}^{2}}\right]\right\} \cdot \exp \{j 2 \pi v(x \cos \theta+y \sin \theta)\}
$$

Where $\theta$ is the direction of the fluctuation of the Gabor function, $\sigma_{x}^{2}$ and $\sigma_{y}^{2}$ are the scales, $u_{x}$ and $u_{y}$ are the mean of the Gaussian function which are used to describe the position of the center of the Gabor function, $v$ is the fluctuation frequency. Equation 1 is a two-dimensional Gaussian function modulated by a complex exponential function. The responses of real part and the imaginary part are the same expect the difference of $\pi / 2$ in phase. Here we shall only use the real part of the Gabor function which is defined as:

$$
g_{\theta}(x, y)=\exp \left\{-\frac{1}{2}\left[\frac{\left(x-u_{x}\right)^{2}}{\lambda \sigma_{x}^{2}}+\frac{\left(y-u_{y}\right)^{2}}{\lambda \sigma_{y}^{2}}\right]\right\} \cdot \cos \{2 \pi v(x \cos \theta+y \sin \theta)\} \text { (2) }
$$

When the fluctuation direction $\theta$ in turn takes $\mathrm{n}$ values equally from 0 to $2 \pi$, the direction of the edge point can be estimated by the $\mathrm{n}$ Gabor functions. Fig. 2 shows the side view and the top view of the two-dimensional Gabor function.

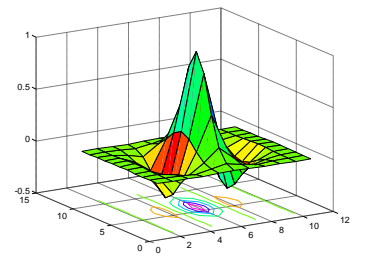

(a) side view

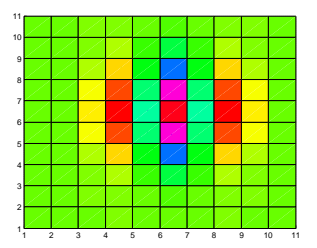

(b) Top view
If $m(x, y)$ is the edge intensity matrix, inner product of the Gabor function in the $\theta_{i}$ direction and the edge strength matrix is defined as:

$$
K_{\theta_{i}}(x, y)=\left\langle g_{\theta_{i}}(x, y), m(x, y)\right\rangle
$$

Where $m(x, y)$ is the edge strength matrix that is a small widow centered at a point $(\mathrm{x}, \mathrm{y})$. Through calculating the inner products of $\mathrm{m}(\mathrm{x}, \mathrm{y})$ by the $\mathrm{n}$ Gabor functions, we obtain $\mathrm{n}$-dimensional vector: 


$$
K(x, y)=\left(K_{\theta_{1}}(x, y), K_{\theta_{2}}(x, y), \cdots, K_{\theta_{n}}(x, y)\right)
$$

If $K_{\theta_{i}}(x, y)$ is the largest component in the n-dimensional vector, the orientation of $g_{\theta_{i}}(x, y)$ is considered as the edge direction. The direction of each edge point is obtained by this method. From the psychology of perception[7] and experimental experience, $\mathrm{N}$ takes 16 , i.e. when the difference of the angle of two-pixel points is $22.5^{\circ}$ that the two points are generally considered to have the same orientation. Because of symmetry, Gabor filters in eight different directions satisfy the condition.

\section{EXTRACTION OF THE CANDIDATE LINE SEGMENT}

After achieving the information of the edge points, the edge points need to be connected or grouped into meaningful segments. The processes of extraction of candidate line segments include two steps: the generation of line-support regions and the rectangular approximation of regions.

\section{A. Line-support regions}

The orientation of the edge point is calculated by the Gabor filter, then the points which share the same angle up to a certain tolerance form a region, and the growing region is defined as the line-support region. The direction of the line segment is the important information for linear feature extraction and detection and recognition of the linear targets. Usually the average direction of the set of pixels [14] is considered as the direction of the line segment. Fig. 3 illustrates the procedure of the region growing algorithm.

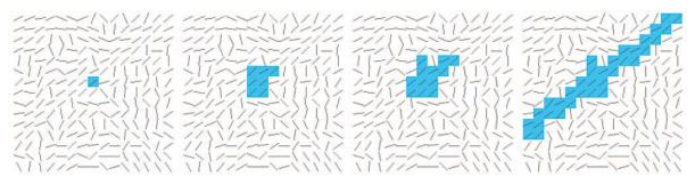

Fig.3. Region growing process of the aligned points

When a seed point is selected, the growing region starts with the seed point and the initial region angle is set to the seed point orientation. Then, the pixels adjacent to the region are tested and judged to satisfy the equation 5.

$$
\operatorname{abs}\left(\operatorname{ang}_{i+1}-\arctan \left(\frac{\sum_{i} \sin \left(\text { ang }_{i}\right)}{\sum_{i} \cos \left(\text { ang }_{i}\right)}\right)\right)<\varepsilon
$$

Where $a n g_{i}, a n g_{i+1}, \varepsilon$, respectively ,represent the angle of the current region, the angle of the next tested pixel and the threshold of the region growing. The pixels satisfied with the function are added to the region. At each iteration, the region angle is updated to the orientation of the region's pixels, defined as follows:

$$
\arctan \left(\frac{\sum_{i} \sin \left(a n g_{i}\right)}{\sum_{i} \cos \left(a n g_{i}\right)}\right)
$$

The process is repeated until no new point can be added.

Edges are usually defined as local rapid changes in some image feature, such as luminance or texture. The rapider the image gray value of the pixels changes in intensity, the more likely the pixels belong to edges. In order to quickly form an effective growing region, reduce the cost of time of the linear feature extraction in SAR image, the edge points detected by ROEWA operator are first in descending order according to the intensity, and then the points are selected as seed point of region growing in turn from the list. In addition, when an edge point is added to a region, it is marked and never visited again.

\section{B. Rectangular approximation and geometric correction}

The intensity variation representing a local edge is often spatially distributed over an extended area due to complex scene lighting conditions interacting with scene surfaces [14]. Besides, the growing regions stated above are usually the wide and irregular shape. So, in most practical situations, the line-support region is often associated with a rectangle approximation. The rectangular approximation is determined by its endpoints and its width or equivalently, its center, angle, length, and width. Its rectangular approximation as shown in Fig.4 includes all of these parameters.

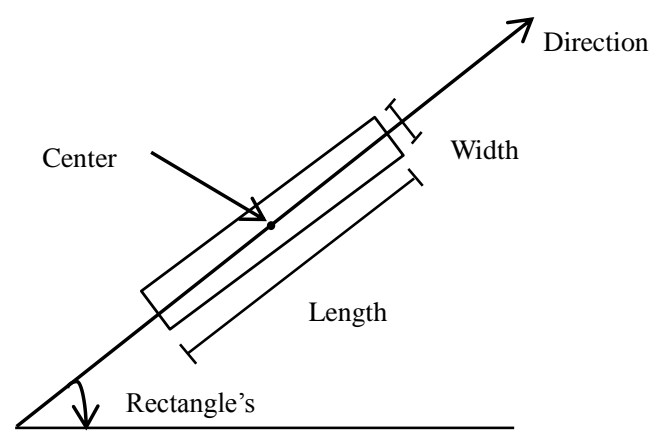

Fig.4the rectangular approximation of regions

In the growing region, the center of mass is used to select the center of the rectangle, and the principal inertia axis to select the rectangle orientation. Then the length and the width are chosen in such a way as to cover the linesupport region. The rectangular approximation is the bounding box of the line-support region, which covers the whole line-support region. So the rectangle inevitably contains some non-edge points. This will result in inaccurate line segment extracted from the rectangle. Thus geometric corrections are needed for the rectangle to exclude the non-edge points. For example, rectangular length, width, etc. should be adjusted when the pixel-density of edge points inside the rectangle is less than the threshold [6]. After geometric correction, line segments need to be validated.

\section{LINE SEGMENT VALIDATION}

The aforementioned processes of edge detection, edge direction estimated by Gabor filter, region growing and rectangular approximation are pixel-level operating. In order to reduce the false detection of the line segment, simultaneously to exclude a large number of unordered and insignificant short line segments and to reduce the burden of 
subsequent calculations, it is necessary to validate the line segments (rectangular approximation) to obtain meaningful ones on the high-level processing.

In order to validate candidate line segments and get the meaningful segments, Desolneux applies the Helmholtz principle [8] to analyze and judge the geometric structure grouped by the Gestalt theory that the structure is a significant image event or not according to the false alarm threshold in statistical hypothesis model. The [8] describes if the expectation in an image of the number of occurrences of the structure which is grouped by points with a characteristic in common (e.g. the same direction) is less than $\varepsilon$ (false alarm threshold) in a uniform random distribution of the background, the structure is called the $\varepsilon$ meaningful image event. NFA ${ }^{[5]}$ (the number of False alarms) as defined in the equation 7 gives the mathematical expression to describe the meaningful rectangle in quantitation. If $N F A(r) \leq \varepsilon$, the rectangles are $\varepsilon^{-}$ meaningful.

$$
N F A(r, x)=N^{5} \bullet b(n(r), k(r), p)
$$

Where $N^{5}$ is the number of the potential rectangles in an $\mathrm{N} \times \mathrm{N}$ image, $b(n, k, p)$ represents that the pixels in random conditions satisfy the binomial distribution, the equation is:

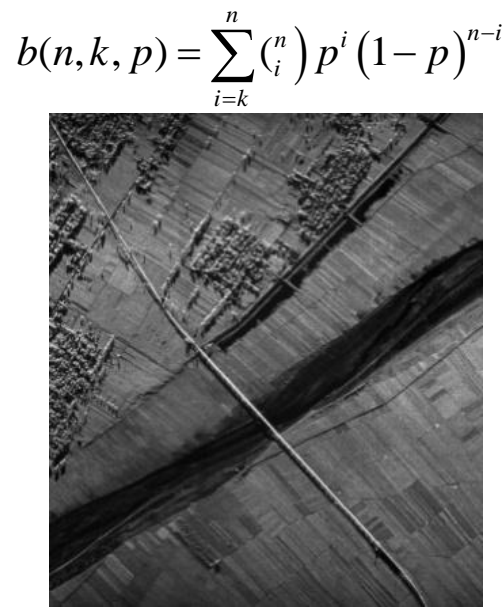

(a) SAR Image
Where $\mathrm{n}$ is the number of pixels in the rectangle, $\mathrm{k}$ is the number of the aligned pixels, $p$ is probability of the point that is aligned with a rectangle, which is decided by the perspective of the region growing. The smaller the value of NFA, the more meaningful the rectangle is.

For high-density texture region, the directions of extracted line segments are messy, disorderly, and isotropic, that validation can well remove these unwanted line segments.

\section{EXPERIMENTAL RESULTS AND ANALYSIS}

To validate the effectiveness and the wideness of the proposed algorithm, two high-resolution SAR images are tested in this letter. Fig. 5 (a) is a SAR image containing a bridge .The image has $2-\mathrm{m}$ resolution and a size of 1316 by 1577 pixels. In the image a large number of buildings, trees and other coverings are in the upper left corner; the river stretches across the middle; the bridge is across the river. Here the phase grouping is applied to extract the linear features compared with the proposed algorithm. Fig. 5 (b) shows the effect by the algorithm of phase grouping [15]. Fig.5 (c) shows the effect by the algorithm proposed in this article. In this experiment, the Gabor filter is selected in eight different directions and the window size of the Gabor filter is set to a size of 11 by 11 pixels. The threshold of the region growing is set to $22.5^{\circ}$. The tolerance false alarm $\varepsilon$ is set to 1 in the validation process.

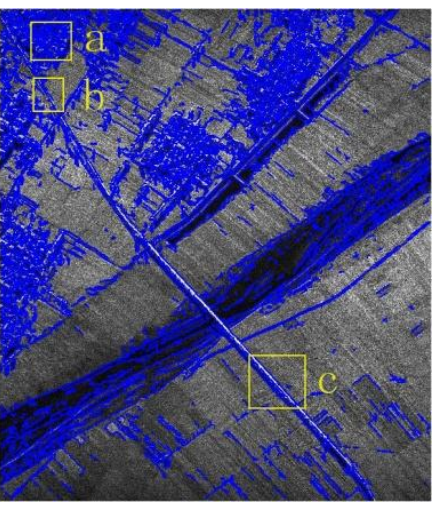

(b) phase grouping

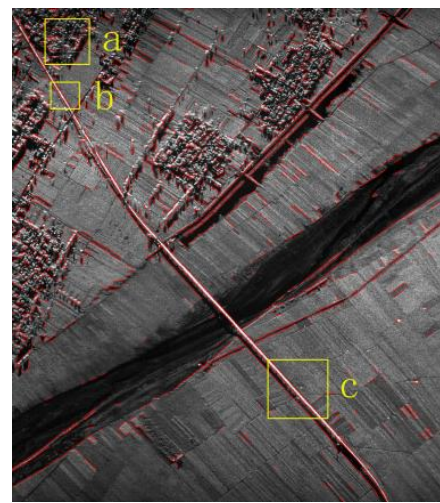

(c) the proposed algorithm

Fig.5.Linear feature extraction of the bridge in the SAR image

In order to illustrate the superiority of this method, here chooses three slices on the same position in the experimental results of the two algorithms, which are labeled with a, b, c three yellow boxes above as shown in Fig. 5 (b) and Fig. 5 (c). Fig. 6 shows the three groups of the slices (each group, the first is the result of the phase grouping; the second is the result of the algorithm in this article).

Comparing the two results of the two algorithms, in the high edge density texture area, the result using phase grouping has many fragmented, short, disordered line segments which are not meaningful. However these short line segments can be considered as well isotropic zones in the validation of the proposed algorithm and so most of them are removed as shown in Fig.6 (a).Besides the proposed algorithm can get grouped and form complete, meaningful line segments as shown in Fig.6 (b). The proposed algorithm makes use of information of the intensity and the direction of the pixels better, so it is able to extract line segments in low contrast regions, as shown in Fig. 6 (c). Considering the cost of time, the algorithm of phase grouping takes about $11 \mathrm{~S}$ (operating environment compatible PC CPU3.1GHz, 1.75G memory, Windows XP operating system, $\mathrm{VC}++$ platform). The proposed algorithm using the method introduces the method stated in 4.1 can speed up the region growing. In the same operating environment, the linear feature extraction by the proposed algorithm has faster processing speed and needs about $7 \mathrm{~s}$ in this image. 


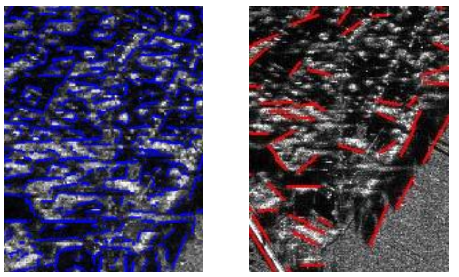

(a) group of slices 1

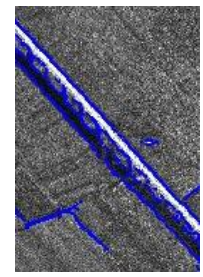

(b) group of slices 2

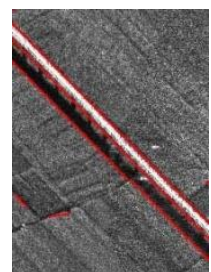

Fig.6. Comparisons of the two methods

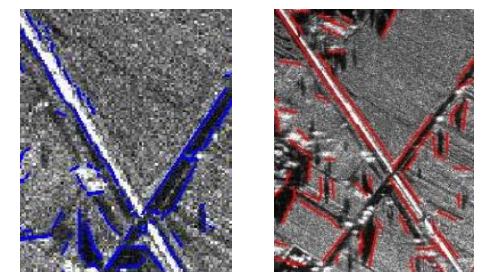

(c) group of slices 3
Meanwhile, in order to show the wideness of the algorithm, the proposed algorithm is applied to the SAR image which contains the runway and has the size of 937 by 737 pixels as shown in Fig. 7(a). From the experimental result as shown in Fig. 7(b), the runway is well extracted. However it only takes about $3 \mathrm{~s}$.

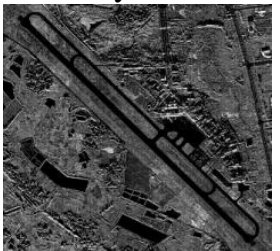

(a) the original image

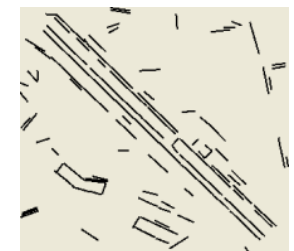

(b) the linear features extracted
Fig.7 . Linear feature extraction of the artificial airport in the SAR image

\section{CONCLUSIONS}

In this article, a new linear feature extraction method based on modified LSD algorithm is described for SAR images. First, ROEWA operator is used to extract edges, and Gabor filter is used to estimate the direction of the edge points accurately; Second, the pixels which share the same direction are grouped and form line-support regions, and then the line-support regions are associated with a candidate line segment (actually, a rectangle) through parametric representation; Finally, calculate the NFA of the rectangle and modify the rectangle to get the meaningful one, at last extract line segments. Some experiments of the linear feature extraction in SAR image have been shown. The results prove that the proposed method can accurately and fast extract linear features in the SAR image. In addition, compared with the phase grouping, the proposed method has stronger antiinterference ability, faster computing speed and has great use in the applications.

\section{ACKNOWLEDGMENT}

I am grateful to Liu Tao for helpful discussions and thank anonymous reviewers for their very helpful and constructive comments on the manuscript.

\section{REFERENCES}

[1] WANG Cheng, WANG Run-sheng. Line Extraction for SAR Image [J]. ACTA ELECTRONICA SINICA : 2003, 31(6):816-820.

[2] CHEN Si, YANG Jian, SONG Xiaoquan. Extraction of linear features in SAR images using grouping and fitting [J]. J T singhua Un iv : Sci \& Tech, 2011,51 (2) : 166-171
[3] Cardoso L A. Computer Aided recognition of man-made structures in Aerial photographs [D]. Naval Postgraduate School, Monterey, USA, 1999.

[4] Venkateswar V, Chellappa R. Extraction of straight lines in aerial images [J]. IEEE Transacations on Pattern Analysis and Mmachine Intelligence, 1992, 14(11): 1111-1114.

[5] Rafael Grompone von Gioi, Jérémie Jakubowice, Jean-Michel More et al. LSD : A Fast Line Segment Detector with a False Detection Control[J]. IEEE TRANSACTIONS ON PATTERN ANALYSIS AND MACHINE INTELLIGENCE, 2010, 32(4):722-732.

[6] R.Grompone von Gioi, J. Jakubowicz, J.M. Morel, and G. Randall, LSD: A Line Segment Detector. IEEE Trans. on PAMI, 19 Dec. 2008

[7] Burns J B, Hanson A R, Riseman E. Extracting straight lines [J]. IEEE Transactions on Pattern Analysis and Machine Intelligence, 1986, 8(4):425-455.

[8] A.Desolneux, J.M.Morel. Meaningful alignments. Int' 1 J. Computer Vision, 2000, 40 (1) : 7-23.

[9] A.Desolneux,L.Moisan,J.M.Morel. From Gestalt Theory to Image Analysis, A Probabilistic Approach [J]. Springer, 2008.

[10] Fjortof R, Lopes A, Marthon P, et al. An optimal multiedge detector for SAR image Smentstion [J].IEEE Transactions on Geoscience and Remote Sensing, 1998, 36 (3) : 793802.

[11] Daugman J G. Uncertainty relation for resolution in space, spatial frequency, and orientation optimized by twodimensional visual cortical filters [J]. Journal of the Optical Society of America, 1985, 2(7):1160-1169.

[12] Frost V S, Stiles J A, Shanmugan K S, et al. A model for radar images and its application to adaptive digital filtering of multiplicative noise [J]. IEEE Transactions on Pattern Analysis and Machine Intelligence, 1982, 4 (2):157-165.

[13] Kong Ying-yin ZHOU Jianjiang. A new edge detection method for SAR Image based on ROEWA and gabor filter[J].Journal of Optorlectrosics laser.2010,21 ( 8 ) : 1257-1263

[14] J. BRIAN BURNS, ALLEN R. HANSON, MEMBER. Extracting Straight Lines[J], IEEE TRANSACTIONS ON PATTERN ANALYSIS AND MACHINE INTELLIGENCE, 1986,8(4):425-456

[15] Jia Chengli. Research of extraction of the road and airport in SAR image [D].Changsha : National University of Defense Technology.2006 